\title{
Pick and Place Automation
}

\author{
Akash Shirsath $^{1}$,Abhay Bhavsar ${ }^{2}$, Lalit Chaudhari ${ }^{3}$, Roshan Pardesi ${ }^{4}$, Atul Barhate ${ }^{5}$ \\ 1,2,3,4,5 Electrical Engg dept, GF's Godavari college of engineering, Jalgaon, India
}

\begin{abstract}
Most of the industry sector in India was found to be using manual methods for finished product, material transfer and picking and placing of products. This consumed time and reduced productivity. This project is for automation of pick and place of components. There is a lot of movement of object under manufacturing before it is picked for dispatch to market or another company. All hardwiring on machines has been now reduced due to PLC programmable logic controller. Biggest advantage is that logic of the machine can be changed without much alteration on the electrical side. HMI, that is human machine interface is used in the system to start the operation or stop it. The idea is to have sensors to sense objects and indicate to PLC. Program in PLC will move the object to location, pick the object and bring it to predefined location using robotic arm. Automation involves electronic, electrical and mechanical components. Accuracy, repeatability \& productivity of the manufacturing process was the main driving force behind the development of Automation Products. Proposed work is for Automation of Pick \& Place of the components. There is a lot of movement of the object under manufacturing before it is picked for dispatch to the market or before it is sent to another shop within the production company. Before automation was integrated into manufacturing, all these movements were either done manually or electromechanically. This consumed time and reduced the productivity. Also, it leads to a lot of inaccuracies due to human fatigue. There are very risky areas of machines which can cause grave human injury. In a fiber winding tension control system unstable tension leads to a strength loss of 20$30 \%$ of the fiber wound component. Automation overcomes all these drawbacks in industrial processes.
\end{abstract}

\section{I -INTRODUCTION}

\section{A} pick and place Automation is the one which is used to pick up an object and place it in the desired location. Pick and Place automation speeds up the process of picking parts up and placing them in new location for assembly or next production cycle. Pick and place are special purpose machines designed to save production time and cost. Pick and place machines speed up the production rate. Mechanical automation has offered more elevated amounts of profitability, productivity, and quality and in addition diminishment to cost on the business. In many Industries, the way towards expanded efficiency is through expanded mechanization process and control. Pick and place automation frameworks will build profitability, productivity and quality control. Amid operation, automation can be controlled to oblige more work, and even work past working hours. With the coming of mechanical technology, arduous and tedious work in modern plant and production lines have made straightforwardness for labourers to finish the operation. These specialists are doled out to control or control these automations as opposed to obliging them to convey the assignment independent from anyone else. This control is made simple with the utilization of Ardunio. The Ardunio is currently utilized as a part of each presentday generation procedures to naturally control each part of the operation without human intercession. These days, automations are progressively being coordinated into working assignments to supplant people particularly to perform the dreary undertaking. As a rule, apply autonomy can be isolated into two regions, modern and administration mechanical technology. These automation 


\section{International Journal of Innovations in Engineering and Science, www.ijies.net}

arms can be independent or controlled physically and can be utilized to perform an assortment of errands with extraordinary exactness. The mechanical arm can be settled or portable (i.e. wheeled) and can be intended for modern or home applications The deciding aftereffect of this anticipate will be a completely practical and exact pick and place framework that can bundle with high repeatability. it then goes into examination of key viewpoints in this anticipate and catches up with points of interest of the outline contemplations and the procedure behind the framework. What's, taking everything into account, condenses the monetary examination of the framework outlined and its advantages, and proposals. Automation as a technology is concerned with the use of mechanical, electrical, electronic and computer-based control systems to replace human beings with machines, not only for physical work but also for the development of information processing. Industrial automation, which started in the eighteenth century as fixed automation has transformed into flexible and programmable automation in the last 15 or 20 years. Computer numerically controlled machine tools, transfer and assembly lines are some examples in this category.

\section{II -NEED OF THE PROJECT}

Automation involves electronic, electrical and mechanical components. Accuracy, repeatability \& productivity of the manufacturing process was the main driving force behind the development of Automation Products. Proposed work is for Automation of Pick \& Place of the components. There is a lot of movement of the object under manufacturing before it is picked for dispatch to the market or before it is sent to another shop within the production company. Before automation was integrated into manufacturing, all these movements were either done manually or electromechanically. This consumed time and reduced the productivity. Also, it leads to a lot of inaccuracies due to human fatigue. There are very risky areas of machines which can cause grave human injury. In a fiber winding tension control system unstable tension leads to a strength loss of $20-30 \%$ of the fiber wound component. Automation overcomes all these drawbacks in industrial processes. everything into account, condenses the monetary examination of the framework outlined and its advantages, and proposals. Automation as a technology is concerned with the use of mechanical, electrical, electronic and computer-based control systems to replace human beings with machines, not only for physical work but also for the development of information processing. Industrial automation, which started in the eighteenth century as fixed automation has transformed into flexible and programmable automation in the last 15 or 20 years. Computer numerically controlled machine tools, transfer and assembly lines are some examples in this category.

\section{III -OBJECTIVES}

The technological objectives of PICK-PLACE are derived from the analysis of the requirements of pickand-package scenarios in Warehouses and Distribution Centers, and answer to the technology gap that represents a market barrier.

Flexible product handling to deal with a variety of products: Technological objective

* To develop a new generation of multifunctional grippers to handle products of different morphology, weight and rigidity and that are able to reach difficult to access target positions.

* To develop reactive grasp-planning algorithm based on cognitive capabilities and allows the robot to effectively grasp different objects.

* Robust and efficient bin-picking solution based on object pose identification and fast and safe robot path planning.

*Dynamic planning to deal with outbound boxes configuration:

* Human and robot affordance aware dynamic package planning for mono and multireference configurations. 1.3.3 Collaborative workspace to deal with high efficiency and high availability of the solution

* Dynamic robot planning based on cognitive capabilities exploiting perceived monitoring and human activity.

* Reliable environment perception system and strategies for safe collaborative scenarios based on Speed and Separation Monitoring combined with Power and Force Limiting.

\section{IV- INDUSTRIAL OBJECTIVES}

To increase the pick-and-package global performance in terms of flexibility, dependability and error reduction.

Improvement of the working conditions of operators by a proper layout design and task allocation between worker and automation.

To control the displacement of the automation so that the arm can be used to pick and place the elements from any source to destination.

\section{V- LITERATURE REVIEW}




\section{International Journal of Innovations in Engineering and Science, www.ijies.net}

M.Cianctietti, A.Arienti, B.M.Follador, B.Mazzalai, P.dario they get inspired by the Octopus to and make an interesting model in automation due to its high dexterity, variable stiffness and very complex behavior. In this experiment they study the key features and patterns of movement of Octopus arm and this features and patterns and patterns of movement are that is elongation, shortening, bending and reaching etc. used for guide the movement of actuator. They conclude that the concept proposed for the mechanism at the base of the automation inspired to the Octopus muscular hydrostat where successfully implemented on mock-ups and the corresponding models have been modified and validate.

Ravi Kumar Mourya, Amit Shelke, Saurabh Satpuite, Sushant Kakade, Monoj Botre have main objective of their project are to design and implement a four DOF pick and place automation. To determine the end effectors position and orientation, theoretical analysis of inverse kinematics are carried out. Ansys software is used for FE Analysis. Prof. S.N.Teli, Akshay Bhalerao, Sagar Ingole, Mahesh Jagadale. This project aims to design and fabricate the pneumatic arm for pick and place of cylindrical objects. They conclude that arm is controlled by manually flaw control and direction control valve. Arm rotation and movement is done by pneumatic cylinder using helical slot mechanism. Total arm weight is $25 \mathrm{~kg}$. The model is expected to lift at least $10 \mathrm{~kg}$ weight. S.Premkumar, K.Surya Varman, R.Ballamurgan, Experimental aim is to collaborate the gripper mechanism and vacuum sucker mechanism working in single pick and place automation.

These automation can perform tasks like gripping, sucking, lifting, placing, releasing, in a single automation arm. It will reduced the cycle time, Ideal time, cost of operation, space consumption. It is user friendly and effectively used in glass handling system. S.C.Gutierrez, R.Zotovic, M.D.Navarra, M.D.Meseguer. Their purpose of work is to manufacture a light weight automation with a low cost budget. They conclude that to avoid negative influence on the total weight of the arm, the plastic material reinforced with fiber is used and vacuum infusion man process is used for manufacturing. Local reinforced elements must be included during construction of arm shell. The mast light gear reducer, harmonic drive types are usedbut because of lack of alignment causes disassembly of gear package to avoid these flexible couplings are required. Gabrielle J.M. Tuithaf, Just L.Harder. Current automations are not safe for interaction with humans, especially for children therefore safe four DOF automation is develop.
M.Pellicciari, G.Berselli, F.Leali, A.Verganana. the method for reducing the total energy consumption of pick and placed automations. It is seen that blowing down an operation as much as possible is not always beneficial. Energy consumption of given operation as a function of the task execution time. Future work includes improvement of the motor model, development of online programming algorithms. Analysis of speed, distance, load lifted by arm is done to know its performance. This automation expected to overcome the problem such as placing or picking object that is away from the user, pick and place hazardous object fast and easily.

John Iovine, in this book various aspects of designing a automation is described. It deals with different types of Arm design, controlling techniques, vehicle design etc. ER. Rajput, in this book the operation and control of automation is discussed. Arduino cookbook, in this book details and methods of interfacing hardware components such as Stepper motor and UNO Aurdino is been discussed.

The other references listed in the references section discusses similar concepts inits various fields such as pick and place identification and segregation, automation for surveillance, pick and place automation controlled using Aurdino etc.

\section{VI- SYSTEM DESIGN}

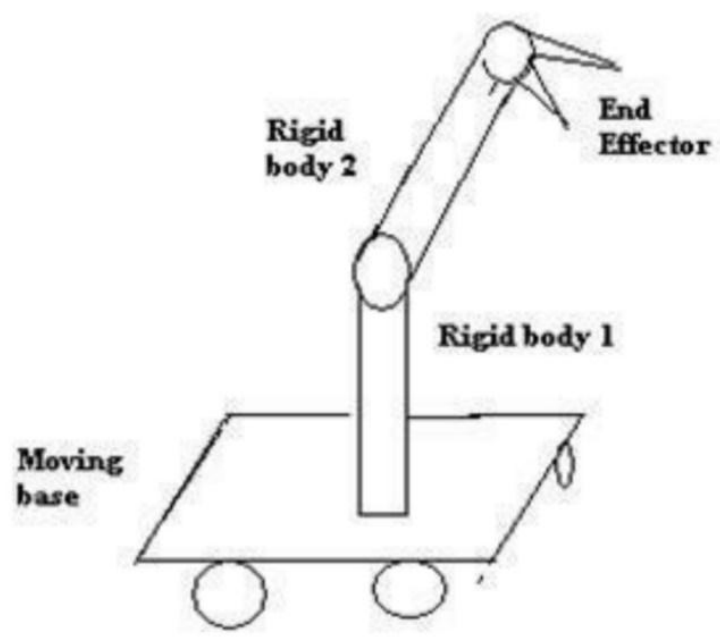

Fig 3- Black Diagram of Proposed Work

Pick and place automation systems are often part of a larger typically pick up components and place them into the fixture for the assembly process, or they are used to pull components off of the system and position them for packaging or for sending them to the next stage in the assembly process. Pick and place automation systems help maximize the efficiency of any automated assembly 


\section{International Journal of Innovations in Engineering and Science, www.ijies.net}

system by reducing labor costs and increasing production throughput. Dial-X Automated Equipment, Inc. has the experience and flexibility to meet your exact pick and place system needs.

\section{Parts of Pick \& Place Mechanism}

Rover: It is the main body of the robot consisting of several rigid bodies like a cylinder or a sphere, joints and links. It is also known as a manipulator.

End Effector: It is the body connected to the last joint of the rover which is used for the purpose of gripping or handling objects. It can be an analogy to the arm of a human being.

Actuators: They are the drivers of the robot. It actually actuates the robot. It can be any motor like servo motor, stepper motor or pneumatic or hydraulic cylinders.

Sensors: They are used to sense the internal as well as the external state to make sure the robot functions smoothly as a whole. Sensors involve touch sensors, IR sensor etc.

Manipulato: The manipulator is the entire mechanism of the robot that provides movement of any degree of freedom. The joints to this robotic manipulator are the movable components, which enables relative motion between the adjoining links. Further they consists of base, arm and gripper.

Grippers: The gripper is similar to the human hand. Just as the hand grasps the tool to perform the work, similarly the gripper secures the robot's work piece while the operation is being performed. As the grip strength and nature vary with respect to objects being gripped, hence shape of the gripper is determined by the task it has to perform.

Controller: It is used to control the actuators based on the sensor feedback and thus control the motion of each and every joint and eventually the movement of the end effector.

Mechanical: It is the most basic and least sophisticated type. For this reason it has been in use from the times when programmable devices did not exist. The purpose of robot arm control was to maintain the dynamic response of the manipulator as per the pre-specified objective through mechanical linkage and cam.

Hydraulic type system: Pascal's law is the basis of hydraulic drive systems and uses pistons and valves to control the movement. This type of controller is used for robots that must move large or heavy loads, because such loads rarely need to be placed with extreme accuracy, e.g. stacking might be task included in this group.

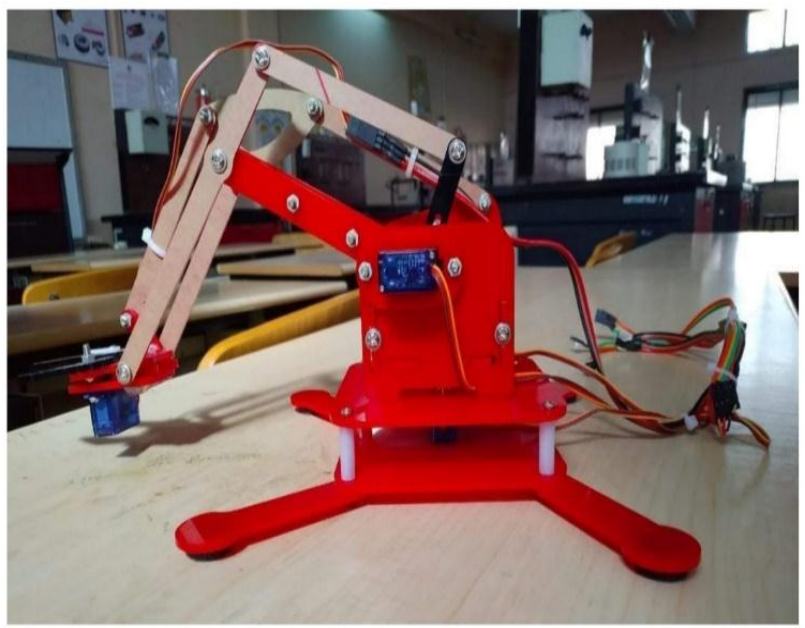

Electrical: This type of control is convenient, clean, quiet, reasonably fast, and used on nearly all assembly robots that require close tolerance accuracy. Therefore this system is most widely used today. Programmable controller microcomputer type, micro process based and minicomputer based controller are used to store instructions to carry out operations.

\section{VII - CONCLUSION}

The goals of this anticipate has been accomplished which was building up the equipment and programming for a Bluetooth controlled automated arm. From perception that has been made, it unmistakably demonstrates that its development is exact, and is anything but difficult to control and easy to understand to utilize. The mechanical arm has been created effectively as the development of the robot can be controlled. This automated arm control strategy is relied upon to conquer the issue, for example, putting or picking protest that far from the client, pick and place risky item in a quick and simple way.

\section{Advantages}

\section{i. Increased efficiency}

Industrial automation can complete certain tasks faster and more efficiently than humans as they are designed and built to perform them with higher accuracy. This combined with the fact they are used to automate processes which previously might have taken significantly more time and resource results in the use of 


\section{International Journal of Innovations in Engineering and Science, www.ijies.net}

industrial automation to increase the efficiency of production

\section{ii. Improved quality}

Given their higher levels of accuracy, industrial automation can be used to produce higher quality products which result in the reduction of time required for quality control and ensures that standards of quality are adhered to.

\section{iii. Increased profitability}

The results of introducing industrial robots can only ensure higher profitability levels with lower cost per product as by increasing the efficiency of your process, reducing the resource and time required to complete it whilst also achieving higher quality products, introducing industrial automation save money in the long run.

\section{iv. Improved working environment}

Some tasks are deemed as too dangerous or laborious and repetitive for humans to carry out and so instead automation can perform these tasks instead. Working conditions, therefore, can be vastly improved as well as the safety within factories and production plants by introducing industrial automation. They are faster and can get the work done in seconds compared to their human counterparts.

v. They are flexible and have the appropriate design.

vi. They are accurate.

vii. They increase the safety of the working environment and actually never get tired.

\section{Disadvantage}

\section{i. Capital cost}

Implementing industrial robots can incur a fairly high capital cost however, they do prove highly effective and bring a positive ROI. This investment allowance means that businesses can deduct qualifying capital expenditure from its taxable profits to spend on machinery, tools and other business equipment.

\section{Future scope}

Future enhancement can include further improvement that is by adding 360 degree rotary servo motor and making it more stable. Setup can be modified that will pick more weight compared to present model.

Ultrasonic sensor can even be placed on the arm so that it can detect and simultaneously pick the object and keep it on other place.

The programmed for pick and place operation can be made versatile and more efficient by providing the feedback and making it to work on own than any human interventions. It can be made possible by image processing tool interfaced with this Arduino. The features that can be added on to improve its efficiency, make it operate on its own thought without any human intervention are line follower, wall hugger, obstacle avoider, metal detector, bomb diffuser etc.

\section{REFERENCES}

[1] Ahmadi, Reza, and John Mamer. "Routing Heuristics for Automated Pick and Place Machines." European Journal of Operational Research, 117.3 (1999): 533 552 .

[2] B.O.Omijeh, R.Uhunmwangho, M.Ehikhamenle, "Design analysis of a remote controlled pick and place robotic vehicle", International journal of Engineering Research and Development. (Volume 10, Issue 5, May 2014).

[3] Khin Moe Myint, Zaw Min MinHtun, HlaMyoTun, "Position Control Method For Pick And Place Robot Arm For Object Sorting System",International journal of scientific \& technology research, Vol. 5, issue 06, pp. 57-61, 2016

[4] Ashly Baby, Chinnu Augustine, Chinnu Thampi, Maria George, Abhilash A P ,Philip C Jose "Pick and Place Robotic Arm Implementation Using Arduino"- IOSR Journal of Electrical and Electronics Engineering (IOSRJEEE) e-ISSN: 22781676,p-ISSN: 2320-3331, Volume 12, Issue 2 Ver. III (Mar. - Apr. 2017), PP 38-41.

[5] Muhammed Jabir.N.K1, Neetha John, Muhammed Fayas, Midhun Mohan, Mithun Sajeev, Safwan.C.N "Control of Pick and Place Robotic Arm Using an Android Application", International Journal of Advanced Research in Electrical, Electronics and Instrumentation Engineering (An ISO 3297: 2007 Certified Organization) Vol. 4, Issue 4, April 2015.

[6] Design and construction of a pick and place Automation arm Theory By Engr. Rana.M.Shakeel

[7] Industrial Pick and Plce Robtic Arm Using Ardiuno Research Paper by Harish K, mega D, Shaklabori M, Amit K, Publish 2017',Page No(1568-1573).

[8] Development of Pick and place Robot for Industrial application by Vishkha borkar and Professor G.k.Andarkar,(volume:04, Issue:09 September 2017, Page no:347 to 356).

[9] International Jurnal of Advanced Re-search In Electrical ,Electronics and Instrumentation Engineering for Pick and Place design by S.Snil Kumar 9an ISO 3297:2007 Certified Organization Vol.4, Issue 6, June 2015).

[10] Development of Robotic arm using ardiuno by priyamboda Mishra, Riki patel, Trushit Upadhyoya ,Arpan Desai by Journal rstablishedin 2000 and upgraded International Journal in 2013 vol 5, Issue 5 May 2017 (ISSN omline $)=2348-3105$.

[11] Er. R.K Rajput, "robotics and Industrial Automation from S. Chond Publication, In 2014. 